\title{
THE FATOU THEOREM AND ITS CONVERSE
}

\author{
BY \\ F. W. GEHRING
}

1. Introduction. Let $H^{+}$denote the class of functions which are nonnegative and harmonic in the upper half plane $y>0$ and let $H$ denote the class of functions which can be expressed as the difference of two functions in $H^{+}$; obviously $H^{+} C H$. It is well known that a function $u(x, y)$ has the Poisson-Stieltjes representation

$$
u(x, y)=K y+\frac{1}{\pi} \int_{-\infty}^{\infty} \frac{y}{(s-x)^{2}+y^{2}} d \alpha(s),
$$

where $K$ is a constant and

$$
\int_{-\infty}^{\infty} \frac{|d \alpha(s)|}{s^{2}+1}<\infty,
$$

if and only if $u(x, y)$ is in $H$ (see [5]). Hence with each function $u(x, y)$ in $H$ we can associate a function $\alpha(s)$ and a constant $K$ such that (1.1) and (1.2) hold; we can further assume that $\alpha(0)=0$. For each $u(x, y)$ in $H^{+}, \alpha(s)$ is nondecreasing and $K \geq 0$.

In this paper we are concerned with the behaviour of a function $u(x, y)$, in $H$, and its derivatives as $(x, y)$ approaches a point $P$ on the $x$-axis. For convenience we take $P$ to be the origin.

Our starting point is the following pair of results (see [4]).

Fatou Theorem. Suppose that $u(x, y)$ is in $H$ and that $\alpha^{\prime}(0)=A$. Then for each $0<\theta<\pi, u(r \cos \theta, r \sin \theta) \rightarrow A$ as $r \rightarrow 0+$.

The converse of this theorem is not true unless we restrict ourselves to the subclass $H^{+}$. In this case we have the following result.

Loomis Theorem. Suppose that $u(x, y)$ is in $H^{+}$, that $0<a \neq b<\pi$, and that for $\theta=a$ and $\theta=b$,

$$
u(r \cos \theta, r \sin \theta) \rightarrow A
$$

as $r \rightarrow 0+$. Then $\alpha^{\prime}(0)=A$.

The Fatou theorem is an Abelian theorem, the Loomis theorem is the corresponding Tauberian theorem, and the Tauberian condition is the restriction that $u(x, y) \geq 0$.

Presented to the Society, November 25, 1955 under the title The converse of Fatou's theorem sor positive harmonic functions; received by the editors March 2, 1956. 
In $\S 3$ we prove several extensions of the Fatou theorem and in $\S 4$ we present the corresponding converses. The arguments in $\$ 4$ are very simple and make use of a variant of the Wiener Tauberian theorem which is developed in $\$ 2$. Finally in $\$ 5$ we consider an extension of a well known result due to Lindelöf.

2. A Tauberian theorem. In this section we introduce a two dimensional form of the Wiener Tauberian theorem for Stieltjes integrals. We begin with some definitions and notation.

A function $f(x)$ is in $L$ if it is Lebesgue integrable over the real line. For $f(x)$ in $L$ we let

$$
F=\int_{-\infty}^{\infty} f(x) d x, \quad F(y)=\int_{-\infty}^{\infty} f(x) e^{i x y} d x
$$

for all real $y$.

A function $f(x)$ is in $M$ if it is continuous for all $x$ and if

$$
\sum_{n=-\infty}^{\infty} \operatorname{Max}_{n \leq x \leq n+1}|f(x)|<\infty .
$$

Obviously $M \subset L$.

A function $\beta(x)$ is in $V$ if it has bounded variation over each finite interval and if

$$
\int_{n}^{n+1}|d \beta(x)|
$$

is bounded in $n$.

Lemma 1. Suppose that $f(x)$ and $g(x)$ are in L. Then $k(x)=f * g(x)$, that is

$$
k(x)=\int_{-\infty}^{\infty} f(x-y) g(y) d y,
$$

exists p.p. in $x, k(x)$ is in $L$, and $K(y)=F(y) G(y)$. Furthermore $k(x)$ is in $M$ $f(x)$ and/or $g(x)$ is in $M$.

Proof. The first part of this lemma follows from known results. For the last part assume that $f(x)$ is in $M$. Then the fact that $f(x)$ is bounded and continuous allows us to apply the Lebesgue "dominated convergence" theorem to conclude that $k(x)$ is continuous. Next we see that

$$
\begin{aligned}
\sum_{n=-\infty}^{\infty} \underset{n \leq x \leq n+1}{\operatorname{Max}}|k(x)| & \leq \sum_{n=-\infty}^{\infty} \operatorname{Max}_{n \leq x \leq n+1} \int_{-\infty}^{\infty}|f(x-y)||g(y)| d y \\
& \leq \sum_{n=-\infty}^{\infty}\left\{\sum_{m=-\infty}^{\infty} \operatorname{Max}_{n-m-1 \leq x \leq n-m+1}|f(x)| \int_{m}^{m+1}|g(y)| d y\right\}
\end{aligned}
$$


and, inverting the order of summation, we conclude that

$$
\sum_{n=-\infty}^{\infty} \operatorname{Max}_{n \leq x \leq n+1}|k(x)| \leq\left(\int_{-\infty}^{\infty}|g(y)| d y\right)\left(\sum_{n=-\infty}^{\infty} \operatorname{Max}_{n \leq x \leq n+2}|f(x)|\right)<\infty
$$

which completes the argument.

The Wiener Tauberian Theorem for Stieltjes integrals can be stated, using (2.1), as follows (see [3, p. 294]).

Wiener Theorem. Suppose that $f(x)$ is in $M$, that $F(y) \neq 0$ for all $y$, that $\beta(x)$ is in $V$, and that

$$
\int_{-\infty}^{\infty} f(x-y) d \beta(y) \rightarrow B
$$

as $x \rightarrow+\infty$. Then for each $h(x)$ in $M$,

$$
\int_{-\infty}^{\infty} h(x-y) d \beta(y) \rightarrow \frac{B}{F} H
$$

as $x \rightarrow+\infty$.

With the aid of Lemma 1 we obtain the following result.

THEOREM 1. Suppose that $f_{1}(x), f_{2}(x), g_{1}(x), g_{2}(x)$ are in $M$, that $F_{1}(y) G_{2}(y)$ $-F_{2}(y) G_{1}(y) \neq 0$ for all $y$, that $\beta(x), \gamma(x)$ are in $V$, and that

$$
\begin{aligned}
& \int_{-\infty}^{\infty} f_{1}(x-y) d \beta(y)+\int_{-\infty}^{\infty} f_{2}(x-y) d \gamma(y) \rightarrow B, \\
& \int_{-\infty}^{\infty} g_{1}(x-y) d \beta(y)+\int_{-\infty}^{\infty} g_{2}(x-y) d \gamma(y) \rightarrow C
\end{aligned}
$$

as $x \rightarrow+\infty(-\infty)$. Then for each $h(x)$ in $M$

$$
\begin{aligned}
\int_{-\infty}^{\infty} h(x-y) d \beta(y) & \rightarrow \frac{B G_{2}-C F_{2}}{F_{1} G_{2}-F_{2} G_{1}} H, \\
\int_{-\infty}^{\infty} h(x-y) d \gamma(y) & \rightarrow \frac{C F_{1}-B G_{1}}{F_{1} G_{2}-F_{2} G_{1}} H
\end{aligned}
$$

as $x \rightarrow+\infty(-\infty)$.

Proof. We consider the case where $x \rightarrow+\infty$; the case where $x \rightarrow-\infty$ then follows with trivial modifications. Set

$$
\begin{aligned}
& b(x)=\int_{-\infty}^{\infty} f_{1}(x-y) d \beta(y)+\int_{-\infty}^{\infty} f_{2}(x-y) d \gamma(y), \\
& c(x)=\int_{-\infty}^{\infty} g_{1}(x-y) d \beta(y)+\int_{-\infty}^{\infty} g_{2}(x-y) d \gamma(y) .
\end{aligned}
$$


Both $b(x)$ and $c(x)$ are bounded by virtue of the hypotheses, (2.2), and (2.3). Hence with (2.4) and the Lebesgue theorem we conclude that

$$
\begin{aligned}
& b * g_{2}(x)=\int_{-\infty}^{\infty} b(x-y) g_{2}(y) d y \rightarrow B G_{2}, \\
& c * f_{2}(x)=\int_{-\infty}^{\infty} c(x-y) f_{2}(y) d y \rightarrow C F_{2}
\end{aligned}
$$

as $x \rightarrow+\infty$. Moreover, from the Fubini theorem it follows that

$$
\begin{aligned}
& b * g_{2}(x)=\int_{-\infty}^{\infty} f_{1} * g_{2}(x-y) d \beta(y)+\int_{-\infty}^{\infty} f_{2} * g_{2}(x-y) d \gamma(y), \\
& c * f_{2}(x)=\int_{-\infty}^{\infty} g_{1} * f_{2}(x-y) d \beta(y)+\int_{-\infty}^{\infty} g_{2} * f_{2}(x-y) d \gamma(y)
\end{aligned}
$$

and, with $k(x)=f_{1} * g_{2}(x)-f_{2} * g_{1}(x)$, we conclude from (2.6) and (2.7) that

$$
\int_{-\infty}^{\infty} k(x-y) d \beta(y) \rightarrow B G_{2}-C F_{2}
$$

as $x \rightarrow+\infty$. By Lemma $1, k(x)$ is in $M$,

$$
K(y)=F_{1}(y) G_{2}(y)-F_{2}(y) G_{1}(y) \neq 0
$$

for all $y$, and we can apply the Wiener theorem to (2.8) to obtain the first part of (2.5). The second part follows in exactly the same way.

To obtain a more convenient form for our Tauberian theorem we perform a familiar exponential change of variable and shift from the interval $-\infty<x<\infty$ to the interval $0<t<\infty$. (For the details see [3, pp. 295-296].) The analogues for $L, M$, and $V$ are as follows.

A function $f(t)$ is in $L^{\prime}$ if it is Lebesgue integrable over $0<t<\infty$. For $f(t)$ in $L^{\prime}$ we let

$$
F=\int_{0}^{\infty} f(t) d t, \quad F(y)=\int_{0}^{\infty} f(t) t^{i v} d t
$$

for all real $y$.

A function $f(t)$ is in $M^{\prime}$ if $f(t)$ is continuous over $0<t<\infty$ and if

$$
\sum_{n=-\infty}^{\infty} \underset{e^{n} \leq t \leq e^{n+1} .}{\operatorname{Max}}|t f(t)|<\infty .
$$

Again $M^{\prime} \subset L^{\prime}$. Observe that $f(t)$ is in $M^{\prime}$ if $f(t)$ is continuous over $0 \leq t<\infty$ and if, for some $\epsilon>0$,

$$
f(t)=O\left(t^{-1-t}\right)
$$

for large $t$ (see [3, p. 299]). 
A function $\beta(t)$ is in $V^{\prime}$ if it has bounded variation over each finite interval in $0<t<\infty$ and if

$$
\int_{t}^{e t} \frac{|d \beta(s)|}{s}
$$

is bounded for all $t>0$.

THEOREM 2. Suppose that $f_{1}(t), f_{2}(t), g_{1}(t), g_{2}(t)$ are in $M^{\prime}$, that $F_{1}(y) G_{2}(y)$ $-F_{2}(y) G_{1}(y) \neq 0$ for all $y$, that $\beta(t), \gamma(t)$ are in $V^{\prime}$, and that

$$
\begin{aligned}
& \frac{1}{t} \int_{0}^{\infty} f_{1}\left(\frac{s}{t}\right) d \beta(s)+\frac{1}{t} \int_{0}^{\infty} f_{2}\left(\frac{s}{t}\right) d \gamma(s) \rightarrow B, \\
& \frac{1}{t} \int_{0}^{\infty} g_{1}\left(\frac{s}{t}\right) d \beta(s)+\frac{1}{t} \int_{0}^{\infty} g_{2}\left(\frac{s}{t}\right) d \gamma(s) \rightarrow C
\end{aligned}
$$

as $t \rightarrow+\infty(0+)$. Then for each $h(t)$ in $M^{\prime}$,

$$
\begin{aligned}
& \frac{1}{t} \int_{0}^{\infty} h\left(\frac{s}{t}\right) d \beta(s) \rightarrow \frac{B G_{2}-C F_{2}}{F_{1} G_{2}-F_{2} G_{1}} H, \\
& \frac{1}{t} \int_{0}^{\infty} h\left(\frac{s}{t}\right) d \gamma(s) \rightarrow \frac{C F_{1}-B G_{1}}{F_{1} G_{2}-F_{2} G_{1}} H
\end{aligned}
$$

as $t \rightarrow+\infty(0+)$.

We require the following corollary in $\$ 4$.

Corollary 1. Suppose, in addition to the hypotheses of Theorem 2 , that $\beta(t)$ and $\gamma(t)$ are monotone and that $\beta(0)=\gamma(0)=0$. Then

$$
\frac{\beta(t)}{t} \rightarrow \frac{B G_{2}-C F_{2}}{F_{1} G_{2}-F_{2} G_{1}}, \frac{\gamma(t)}{t} \rightarrow \frac{C F_{1}-B G_{1}}{F_{1} G_{2}-F_{2} G_{1}}
$$

as $t \rightarrow+\infty(0+)$.

Proof. If we set $h(t)=e^{-t}$, then $h(t)$ is in $M^{\prime}$ and we conclude, from (2.10), that

$$
\begin{aligned}
& \frac{1}{t} \int_{0}^{\infty} e^{-(s / t)} d \beta(s) \rightarrow \frac{B G_{2}-C F_{2}}{F_{1} G_{2}-F_{2} G_{1}}, \\
& \frac{1}{t} \int_{0}^{\infty} e^{-(s / t)} d \gamma(s) \rightarrow \frac{C F_{1}-B G_{1}}{F_{1} G_{2}-F_{2} G_{1}}
\end{aligned}
$$

as $t \rightarrow+\infty(0+)$. Now (2.11) follows from a familiar Tauberian theorem for the Laplace transform. (See $[8$, p. 192].)

3. Extensions of the Fatou theorem. Here we develop some Abelian results related to the Fatou theorem. It will be convenient to make use of the 
complex notation, writing $u(x+i y)$ for $u(x, y)$ and letting $R(w)$ and $I(w)$ denote the real and imaginary parts, respectively, of the complex number $w$.

For $u(z)$, harmonic, and $n \geq 1$ we let

$$
D_{\psi}^{n} u=\cos \psi \frac{\partial^{n} u}{\partial x^{n}}+\sin \psi \frac{\partial^{n} u}{\partial y \partial x^{n-1}}
$$

denote the $n$th directional derivative of $u(z)$ in the direction $\psi$. Observe that

$$
D_{\psi}^{n} u=R\left(e^{i \psi} \frac{d^{n} f}{d z^{n}}\right)
$$

where $f(z)$ is analytic with $u=R(f)$, and that any linear combination of $n$th derivatives of $u(z)$ can be expressed as $C D_{\psi} u(z)$, where $C$ is a constant and $\psi$ is an appropriate angle.

For $u(z)$ in $H$ we have the Poisson-Stieltjes representation (1.1) which, in complex notation, reduces to

$$
u(z)=K y+\frac{1}{\pi} \int_{-\infty}^{\infty} I\left\{\frac{1}{s-z}\right\} d \alpha(s) .
$$

The Lebesgue theorem and (1.2) allow us to differentiate underneath the integral sign in (3.1) to obtain

$$
D_{\psi}^{1} u(z)=K \sin \psi+\frac{1}{\pi} \int_{-\infty}^{\infty} I\left\{\frac{e^{i \psi}}{(s-z)^{2}}\right\} d \alpha(s)
$$

and, for $n>1$,

$$
D_{\psi}^{n} u(z)=\frac{n !}{\pi} \int_{-\infty}^{\infty} I\left\{\frac{e^{i \psi}}{(s-z)^{n+1}}\right\} d \alpha(s) .
$$

Next for any $c>0$, it follows from (1.2) that

$$
\int_{|s| \geq c} \frac{|d \alpha(s)|}{|s-z|^{2}}=O(1)
$$

as $z \rightarrow 0$ and hence

$$
\begin{aligned}
\int_{|s| \geq c} I\left\{\frac{1}{s-z}\right\} d \alpha(s) & =O(y), \\
\int_{|s| \geq c} I\left\{\frac{e^{i \psi}}{(s-z)^{n+1}}\right\} d \alpha(s) & =O(1)
\end{aligned}
$$

as $z \rightarrow 0$.

We list some formulas which are required later on. 
Lemma $2\left(^{1}\right)$. Suppose that $-1<R(\zeta)<1$, that $0<\theta<\pi$, and that $n \geq 1$. Then

$$
\begin{aligned}
\frac{1}{\pi} \int_{0}^{\infty} I\left\{\frac{1}{s-e^{i \theta}}\right\} s^{\zeta} d s & =\frac{\sin (\pi-\theta) \zeta}{\sin \pi \zeta} \\
-\frac{1}{\pi} \int_{0}^{\infty} I\left\{\frac{1}{s+e^{i \theta}}\right\} s^{\zeta} d s & =\frac{\sin \theta \zeta}{\sin \pi \zeta}, \\
\frac{n !}{\pi} \int_{0}^{\infty} I\left\{\frac{e^{i \psi}}{\left(s-e^{i \theta}\right)^{n+1}}\right\} s^{\zeta} d s & =\frac{(\zeta)_{n} \sin [(\pi-\theta) \zeta-(\psi-n \theta)]}{\sin \pi \zeta} \\
(-1)^{n+1} \frac{n !}{\pi} \int_{0}^{\infty} I\left\{\frac{e^{i \psi}}{\left(s+e^{i \theta}\right)^{n+1}}\right\} s^{\zeta} d s & =\frac{(\zeta)_{n} \sin [\theta \zeta+(\psi-n \theta)]}{\sin \pi \zeta}
\end{aligned}
$$

where we set

$$
(\zeta)_{n}=\zeta(\zeta-1) \cdots(\zeta-n+1)
$$

Proof. (3.6) and (3.8) can be established for $-\pi<\theta<\pi$ by contour integration. (Alternatively, they can be deduced from known Mellin transforms. See [2, pp. 307-308].) Then (3.5) and (3.7) follow from (3.6) and (3.8) by substituting $\theta-\pi$ for $\theta$.

Our first Abelian theorem is the following result.

Theorem 3. Suppose that $u(z)$ is in $H$, that $-1<\delta<1$, and that

$$
\alpha(t)=O(|t| 1+\delta)
$$

as $t \rightarrow 0$. Then for all $0<\theta<\pi, \psi$, and $n \geq 1$,

$$
u\left(r e^{i \theta}\right)=O\left(r^{\delta}\right), D_{\psi} u\left(r e^{i \theta}\right)=\stackrel{n}{O}\left(r^{\delta-n}\right)
$$

as $r \rightarrow 0+$. We can replace " $O$ " by " $O$ " in both the hypotheses and the conclusions.

Proof. We will consider the " $o$ " case. By hypothesis we can select $c>0$ such that

$$
|\alpha(t)| \leq \epsilon|t|^{1+\delta}
$$

for $|t| \leq c$ and by (3.4) we can assume that $\alpha(t)$ is constant outside of this interval. Next we can assume that $K=0$ in (3.1) and (3.2) and, with an integration by parts, we obtain

$$
\begin{aligned}
u(z) & =\frac{1}{\pi} \int_{-\infty}^{\infty} I\left\{\frac{1}{(s-z)^{2}}\right\} \alpha(s) d s, \\
D_{\psi}^{n} u(z) & =\frac{(n+1) !}{\pi} \int_{-\infty}^{\infty} I\left\{\frac{e^{i \psi}}{(s-z)^{n+2}}\right\} \alpha(s) d s .
\end{aligned}
$$

(1) When $\zeta=0$ in (3.5), (3.6), (3.7), (3.8), (4.12) and similarly when $\delta=0$ in (3.13), (4.5), (4.7), the left hand side of each equation is equal to the limit of the right hand side as the relevant variable, $\zeta$ or $\delta$, approaches 0 . 
Then with (3.10) we have

$$
\begin{gathered}
\left|u\left(r e^{i \theta}\right)\right| \leq \frac{2 r \sin \theta}{\pi} \int_{-\infty}^{\infty} \frac{\epsilon|s|^{1+\delta}}{\left|s-r e^{i \theta}\right|^{3}} d s, \\
\left|D_{\downarrow}^{n} u\left(r e^{i \theta}\right)\right| \leq \frac{(n+1) !}{\pi} \int_{-\infty}^{\infty} \frac{\epsilon|s|^{1+\delta}}{\left|s-r e^{i \theta}\right|^{n+2}} d s
\end{gathered}
$$

and the desired conclusions follow immediately since, for $n \geq 1$,

$$
\int_{-\infty}^{\infty} \frac{|s|^{1+\delta}}{\left|s-r e^{i \theta}\right|^{n+2}} d s=r^{\delta-n} \int_{-\infty}^{\infty} \frac{|\sigma|^{1+\delta}}{\left|\sigma-e^{i \theta}\right|^{n+2}} d \sigma=C r^{\delta-n}
$$

where $C=C(\theta, \delta, n)<\infty$.

TheOREM 4. Suppose that $u(z)$ is in $H$, that $-1<\delta<1$, and that

$$
\begin{aligned}
\alpha(t) & \sim A t^{1+\delta} \\
-\alpha(-t) & \sim B t^{1+\delta}
\end{aligned}
$$

as $t \rightarrow 0+$. Then for all $0<\theta<\pi, \psi$, and $n \geq 1$,

$$
\begin{aligned}
u\left(r e^{i \theta}\right) & =u^{*}\left(r e^{i \theta}\right)+o\left(r^{\delta}\right), \\
D_{\downarrow}^{n} u\left(r e^{i \theta}\right) & =D_{\downarrow}^{n} u^{*}\left(r e^{i \theta}\right)+o\left(r^{\delta-n}\right)
\end{aligned}
$$

as $r \rightarrow 0+$, where

$$
u^{*}\left(r e^{i \theta}\right)=(1+\delta) \frac{A \sin (\pi-\theta) \delta+B \sin \theta \delta}{\sin \pi \delta} r^{\delta} .
$$

Proof. This last result follows almost directly from Theorem 3. First set

$$
\alpha^{*}(t)=A t^{1+\delta}, \quad-\alpha^{*}(-t)=B t^{1+\delta}
$$

for $0 \leq t<\infty$; then $\alpha^{*}(t)$ satisfies (1.2) and

$$
u^{*}(z)=\frac{1}{\pi} \int_{-\infty}^{\infty} I\left\{\frac{1}{s-z}\right\} d \alpha^{*}(s)
$$

is in $H$. From (3.11) and (3.14) it follows that

$$
\alpha(t)-\alpha^{*}(t)=o\left(|t|^{1+\delta}\right)
$$

as $t \rightarrow 0$ and hence (3.12) follows from Theorem 3. Finally we see that $u^{*}\left(r e^{i \theta}\right)$ is simply

$$
(1+\delta)\left\{\frac{A}{\pi} \int_{0}^{\infty} I\left(\frac{1}{\sigma-e^{i \theta}}\right) \sigma^{\delta} d \sigma-\frac{B}{\pi} \int_{0}^{\infty} I\left(\frac{1}{\sigma+e^{i \theta}}\right) \sigma^{\delta} d \sigma\right\} r^{\delta}
$$

and (3.13) follows from (3.5) and (3.6) of Lemma 2 with $\zeta=\delta$.

(2) Here and in what follows $\phi(x) \sim C x^{\rho}$ means $x^{-\rho} \phi(x) \rightarrow C$ as $x \rightarrow 0+$. 
Theorem 4 establishes a relation between the behaviour of $\alpha(t)$ near $t=0$ and the behaviour of $u(z)$ near $z=0$ for $|\delta|<1$. (In particular we obtain the Fatou theorem when $\delta=0$ and $A=B$.) It is natural to inquire about the situation when $|\delta|=1$.

The following two results discuss the cases when $\delta=-1$ and $\delta=1$ respectively.

TheOREm 5. Suppose that $u(z)$ is in $H$ and that

$$
\alpha(t)-\alpha(-t) \rightarrow A \pi
$$

as $t \rightarrow 0+$. Then for all $0<\theta<\pi, \psi$, and $n \geq 1$,

$$
\begin{aligned}
u\left(r e^{i \theta}\right) & =u^{*}\left(r e^{i \theta}\right)+o\left(r^{-1}\right), \\
D_{\psi}^{n} u\left(r e^{i \theta}\right) & =D_{\psi}^{n} u^{*}\left(r e^{i \theta}\right)+o\left(r^{-1-n}\right)
\end{aligned}
$$

as $r \rightarrow 0+$, where $u^{*}(x, y)=A y\left(x^{2}+y^{2}\right)$.

Theorem 6. Suppose that $u(z)$ is in $H$ and that the integral

$$
\frac{1}{\pi} \int_{-\infty}^{\infty} \frac{d \alpha(s)}{s^{2}}=A
$$

is absolutely convergent. Then for all $0<\theta<\pi, \psi$, and $n \geq 1$,

$$
\begin{aligned}
u\left(r e^{i \theta}\right) & =u^{*}\left(r e^{i \theta}\right)+o(r), \\
D_{\psi}^{n} u\left(r e^{i \theta}\right) & =D_{\psi}^{n} u^{*}\left(r e^{i \theta}\right)+o\left(r^{1-n}\right)
\end{aligned}
$$

as $r \rightarrow 0+$, where $u^{*}(x, y)=(A+K) y$.

Proofs. First note that Theorem 3 holds for $\delta=-1$; Theorem 5 is then established by an argument similar to the one used in the proof of Theorem 4 .

The hypotheses of Theorem 6 imply that $1 / s^{2}$ is integrable, in the LebesgueStieltjes sense, over the real line. By (3.1),

$$
\frac{u\left(r e^{i \theta}\right)}{r \sin \theta}=K+\frac{1}{\pi} \int_{-\infty}^{\infty} \frac{d \alpha(s)}{\left|s-r e^{i \theta}\right|^{2}} .
$$

For $s \neq 0,1 /\left|\left(s-r e^{i \theta}\right)\right|^{2}$ is bounded by $C / s^{2}$ and converges to $1 / s^{2}$ as $r \rightarrow 0+$, and the first part of (3.16) follows by the "dominated convergence" theorem. The second part follows similarly.

When $u(z)$ is in $H$, there is no significant Fatou theorem with $|\delta|>1$. For on the one hand, $\alpha(t)$ has left and right hand limits at each point and hence the conclusion of Theorem 5 always holds for some value of $A$. On the other hand, Theorem 6 shows us that for $\delta \geq 1$ the behaviour of $u(z)$ near $z=0$ depends upon the values $\alpha(t)$ assumes along the entire real line. For functions in $H^{+}$this last fact is strikingly illustrated in Theorem 11.

4. Extensions of the Loomis theorem. We begin with a result which, for functions in $H^{+}$, is a converse for Theorem 3 (cf. [1, Lemma 1]). 
TheOREM 7. Suppose that $u(z)$ is in $H^{+}$, that $\delta$ is any real number, that $0<a<\pi$, and that for $\theta=a$,

$$
u\left(r e^{i \theta}\right)=O\left(r^{\delta}\right)
$$

as $r \rightarrow 0+$. Then (4.1) holds for all $0<\theta<\pi$ and

$$
\alpha(t)=O\left(|t|^{1+\delta}\right)
$$

as $t \rightarrow 0$. We can replace " $O$ " by " $o$ " in the hypotheses and the conclusions.

Proof. It is not difficult to see that we may take $K=0$ in (3.1). Then, with

$$
C=\frac{\sin \theta}{\sin a} \underset{|\sigma|<\infty}{\operatorname{LUB}}\left|\frac{\sigma-e^{i a}}{\sigma-e^{i \theta}}\right|^{2}<\infty,
$$

we obtain

$$
\begin{aligned}
u\left(r e^{i \theta}\right) & =\frac{1}{\pi} \int_{-\infty}^{\infty} \frac{r \sin \theta}{\left|s-r e^{i \theta}\right|^{2}} d \alpha(s) \\
& \leq \frac{C}{\pi} \int_{-\infty}^{\infty} \frac{r \sin a}{\left|s-r e^{i a}\right|^{2}} d \alpha(s)=C u\left(r e^{i a}\right) .
\end{aligned}
$$

The second part follows since

$$
\frac{\alpha(t)-\alpha(-t)}{t} \leq 2 \int_{-t}^{t} \frac{t}{s^{2}+t^{2}} d \alpha(s) \leq 2 \pi u(0, t)
$$

for each $t>0$.

The next three theorems are converses for Theorem 4 . In these results we assume that $0<a, b<\pi$, that $-1<\delta<1$, and that $m, n \geq 1$. Furthermore we set

$$
\mu=c-m a, \quad \eta=d-n b,
$$

and we adopt the notation (3.9).

TheOREM 8. Suppose that $u(z)$ is in $H^{+}$, that $a \neq b$, and that

$$
u\left(r e^{i a}\right) \sim A r^{\delta}, u\left(r e^{i b}\right) \sim B r^{\delta}
$$

as $r \rightarrow 0+$. Then

$$
\alpha(t) \sim C t^{1+\delta}, \quad-\alpha(-t) \sim D t^{1+\delta}
$$

as $t \rightarrow 0+$, where

$$
\begin{aligned}
(1+\delta) C & =\frac{A \sin b \delta-B \sin a \delta}{\sin (b-a) \delta} \\
(1+\delta) D & =\frac{B \sin (\pi-a) \delta-A \sin (\pi-b) \delta}{\sin (b-a) \delta}
\end{aligned}
$$


Theorem 8 was first proved by Allen and Kerr by a different method (see [1, Theorem III]).

Theorem 9. Suppose that $u(z)$ is in $H^{+}$, that $(b-a) \delta+\eta \neq \equiv 0 \bmod \pi$, and that

$$
\begin{aligned}
u\left(r e^{i a}\right) & \sim A r^{\delta} \\
D_{d}^{n}\left(r e^{i b}\right) & \sim B r^{\delta-n}
\end{aligned}
$$

as $r \rightarrow 0+$. Then (4.4) holds with

$$
\begin{aligned}
(1+\delta) C & =\frac{(\delta)_{n} A \sin [b \delta+\eta]-B \sin a \delta}{(\delta)_{n} \sin \lfloor(b-a) \delta+\eta\rfloor} \\
(1+\delta) D & =\frac{B \sin (\pi-a) \delta-(\delta)_{n} A \sin [(\pi-b) \delta-\eta]}{(\delta)_{n} \sin [(b-a) \delta+\eta]}
\end{aligned}
$$

Theorem 10. Suppose that $u(z)$ is in $H^{+}$, that $(b-a) \delta+R-\mu \not \equiv 0 \bmod \pi$, that $\delta \neq 0$, and that

$$
\begin{aligned}
u\left(r e^{i a}\right) & =O\left(r^{\delta}\right), \\
D_{c}^{m} u\left(r e^{i a}\right) & \sim A r^{\delta-m}, \\
D_{d}^{n} u\left(r e^{i b}\right) & \sim B r^{\delta-m}
\end{aligned}
$$

as $r \rightarrow 0+$. Then (4.4) holds with

$$
\begin{aligned}
(1+\delta) C & =\frac{(\delta)_{n} A \sin [b \delta+\eta]-(\delta)_{m} B \sin [a \delta+\mu]}{(\delta)_{m}(\delta)_{n} \sin [(b-a) \delta+\eta-\mu]}, \\
(1+\delta) D & =\frac{(\delta)_{m} B \sin [(\pi-a) \delta-\mu]-(\delta)_{n} A \sin [(\pi-b) \delta-\eta]}{(\delta)_{m}(\delta)_{n} \sin [(b-a) \delta+\eta-\mu]} .
\end{aligned}
$$

Proofs. The proofs for these three theorems follow along similar lines. We give only the details for Theorem 9.

By (3.4) we can assume that $\alpha(t)$ is constant for large $t$; by (4.6) and Theorem 7 we see that

$$
\alpha(t)=O(|t| 1+\delta)
$$

for small $t$. From these two observations it follows that

$$
\begin{aligned}
& \beta(t)=\int_{0}^{t} \frac{d \alpha(s)}{s^{\delta}} \\
& \gamma(t)=-\int_{0}^{t} \frac{d \alpha(-s)}{s^{\delta}}
\end{aligned}
$$

are defined and that 


$$
\begin{aligned}
& \int_{t}^{e t} \frac{|d \beta(s)|}{s}=\int_{t}^{e t} \frac{d \alpha(s)}{s^{1+\delta}} \leq \frac{\alpha(e t)-\alpha(t)}{t^{1+\delta}}=O(1), \\
& \int_{t}^{e t} \frac{|d \gamma(s)|}{s}=-\int_{t}^{e t} \frac{d \alpha(-s)}{s^{1+\delta}} \leq \frac{\alpha(-t)-\alpha(-e t)}{t^{1+\delta}}=O(1)
\end{aligned}
$$

for all $t>0$. Hence $\beta(t)$ and $\gamma(t)$ are in $V^{\prime}$. Furthermore, by a Tauberian theorem due to Allen and Kerr,

$$
\begin{aligned}
\frac{\alpha(t)}{t^{1+\delta}} \rightarrow C \quad \text { iff } \quad \frac{\beta(t)}{t} \rightarrow(1+\delta) C, \\
\frac{-\alpha(-t)}{t^{1+\delta}} \rightarrow D \quad \text { iff } \quad \frac{\gamma(t)}{t} \rightarrow(1+\delta) D
\end{aligned}
$$

as $t \rightarrow 0+$ (see [1, Lemma 2]).

Once again we can assume that $K=0$ in (3.1) and (3.2). Then with (4.8) we obtain

$$
\begin{aligned}
u(z) & =\frac{1}{\pi} \int_{0}^{\infty} I\left\{\frac{s^{\delta}}{s-z}\right\} d \beta(s)-\frac{1}{\pi} \int_{0}^{\infty} I\left\{\frac{s^{\delta}}{s+z}\right\} d \gamma(s), \\
D_{\psi}^{n} u(z)= & \frac{n !}{\pi} \int_{0}^{\infty} I\left\{\frac{e^{i \psi} s^{\delta}}{(s-z)^{n+1}}\right\} d \beta(s)+(-1)^{n+1} \frac{n !}{\pi} \int_{0}^{\infty} I\left\{\frac{e^{i \psi} s^{\delta}}{(s+z)^{n+1}}\right\} d \gamma(s),
\end{aligned}
$$

and with

$$
\begin{array}{ll}
f_{1}(t)=\frac{1}{\pi} I\left\{\frac{t^{\delta}}{t-e^{i a}}\right\}, & f_{2}(t)=-\frac{1}{\pi} I\left\{\frac{t^{\delta}}{t+e^{i a}}\right\}, \\
g_{1}(t)=\frac{n !}{\pi} I\left\{\frac{e^{i d} t^{\delta}}{\left(t-e^{i b}\right)^{n+1}}\right\}, & g_{2}(t)=(-1)^{n+1} \frac{n !}{\pi} I\left\{\frac{e^{i d} t^{\delta}}{\left(t+e^{i b}\right)^{n+1}}\right\}
\end{array}
$$

we conclude that

$$
\begin{aligned}
r^{-\delta} u\left(r e^{i a}\right) & =\frac{1}{r} \int_{0}^{\infty} f_{1}\left(\frac{s}{r}\right) d \beta(s)+\frac{1}{r} \int_{0}^{\infty} f_{2}\left(\frac{s}{r}\right) d \gamma(s), \\
r^{n-\delta} D_{d}^{n} u\left(r e^{i b}\right) & =\frac{1}{r} \int_{0}^{\infty} g_{1}\left(\frac{s}{r}\right) d \beta(s)+\frac{1}{r} \int_{0}^{\infty} g_{2}\left(\frac{s}{r}\right) d \gamma(s)
\end{aligned}
$$

for $r>0$. By virtue of (2.9), all the functions in (4.10) are in $M^{\prime}$ and, by Lemma 2 and (4.2), we have

$$
\begin{array}{ll}
F_{1}(y)=\frac{\sin (\pi-a) \zeta}{\sin \pi \zeta}, & F_{2}(y)=\frac{\sin a \zeta}{\sin \pi \zeta}, \\
G_{1}(y)=\frac{(\zeta)_{n} \sin [(\pi-b) \zeta-\eta]}{\sin \pi \zeta}, & G_{2}(y)=\frac{(\zeta)_{n} \sin [b \zeta+\eta]}{\sin \pi \zeta},
\end{array}
$$


where $\zeta=\delta+i y$. It follows that

$$
F_{1}(y) G_{2}(y)-F_{2}(y) G_{1}(y)=\frac{(\zeta)_{n} \sin [(b-a) \zeta+\eta]}{\sin \pi \zeta} \neq 0
$$

for all real $y$ and we appeal to (4.6), (4.11), Corollary 1, and (4.9) to complete the proof.

Unfortunately, the restriction that $\delta \neq 0$ is essential in Theorem 10 . For consider

$$
f(z)=(\log z)^{i}=e^{i \log (\log z)}
$$

where, for $z=\rho e^{i \phi}$ and $0<\phi<\pi$, we define

$$
\log z=\log \rho+i \phi .
$$

Now $f(z)$ is analytic and $|f(z)| \leq 1$ in $y>0$. Furthermore it is not difficult to show that for all $0<\theta<\pi$ and $n \geq 1$,

$$
\frac{d^{n}}{d z^{n}} f\left(r e^{i \theta}\right)=o\left(r^{-n}\right)
$$

as $r \rightarrow 0+$. With $u=R(f)+1$ it follows that

$$
\limsup _{r \rightarrow 0+} u\left(r e^{i \theta}\right)-\liminf _{r \rightarrow 0+} u\left(r e^{i \theta}\right)=2 e^{-\pi},
$$

for each $0<\theta<\pi$, and that $u(z)$ satisfies the hypotheses of Theorem 10 with $\delta=0$ and $A=B=0$. However, (4.4) cannot hold for any $C$ and $D$ for otherwise Theorem 4 would contradict (4.13).

Finally consider the situation when $|\delta|=1$. Though Theorem 5 has no converse we can invert Theorem 6 as follows (cf. [7, p. 208]).

TheOREM 11. Suppose that $u(z)$ is in $H^{+}$and that $0<a<\pi$. If

$$
u\left(r e^{i a}\right)=O(r)
$$

as $r \rightarrow 0+$, then the integral (3.15) is absolutely convergent. If

$$
u\left(r e^{i a}\right)=o(r)
$$

as $r \rightarrow 0+$, then $u(z) \equiv 0$.

Proof. By Theorem 7 we see that

$$
\frac{u(0, t)}{t}=K+\frac{1}{\pi} \int_{-\infty}^{\infty} \frac{d \alpha(s)}{s^{2}+t^{2}}=O(1)
$$

as $t \rightarrow 0+$ and, by Fatou's lemma, we conclude that

$$
\frac{1}{\pi} \int_{-\infty}^{\infty} \frac{d \alpha(s)}{s^{2}} \leq \liminf _{t \rightarrow 0+} \frac{1}{\pi} \int_{-\infty}^{\infty} \frac{d \alpha(s)}{s^{2}+t^{2}}<\infty .
$$


With (4.14) we can replace " $O$ " by " $o$ " on the right side of (4.15) and we conclude that $K$ and the left side of (4.16) are equal to zero; hence $\alpha(t) \equiv 0$.

5. An application. Here we use Theorem 9 to obtain an extension of the following result due to Lindelöf (see $[6$, p. 70]).

LindelöF TheOREM. Suppose that $f(z)$ is analytic and bounded in $y>0$, that $0<a<\pi$, and that for $\theta=a$,

$$
f\left(r e^{i \theta}\right) \rightarrow A+i B
$$

as $r \rightarrow 0+$. Then (5.1) holds for all $0<\theta<\pi$.

Lemma 3. Suppose that $u(z)$ is in $H$, that $v(z)$ is a conjugate for $u(z)$, that $-1<\delta<1$, and that

$$
\alpha(t)=O\left(|t|^{1+\delta}\right)
$$

as $t \rightarrow 0$. If $0<a<\pi$ and

$$
u\left(r e^{i a}\right) \sim A r^{\delta}
$$

as $r \rightarrow 0+$, then

$$
\frac{\partial}{\partial r} u\left(r e^{i a}\right) \sim \delta A r^{\delta-1}
$$

as $r \rightarrow 0+$. If $0<b<\pi$ and

$$
v\left(r e^{i b}\right) \sim B r^{\delta}
$$

as $r \rightarrow 0+$, then

$$
\frac{\partial}{\partial r} v\left(r e^{i b}\right) \sim \delta B r^{\delta-1}
$$

as $r \rightarrow 0+$.

Proof. For the first part set $\phi(r)=u\left(r e^{i a}\right)$ for $r>0$. By (5.3), (5.2), and Theorem 3 we see that

$$
\begin{gathered}
\phi(r) \sim A r^{\delta}, \\
\phi^{\prime \prime}(r)=O\left(r^{\delta-2}\right)
\end{gathered}
$$

as $r \rightarrow 0+$, and we conclude that

$$
\phi^{\prime}(r) \sim \delta A r^{\delta-1}
$$

as $r \rightarrow 0+$ by a well known Tauberian theorem due to Hardy and Littlewood (see $[8$, p. 194]). The second part follows similarly.

Observe that Lemma 3 allows us to deduce Theorem 8 from Theorem 9. For the hypotheses (4.3) imply that (5.2) holds, by virtue of Theorem 7, and hence that 


$$
\begin{aligned}
u\left(r e^{i a}\right) & \sim A r^{\delta}, \\
D_{b}^{1} u\left(r e^{i b}\right) & \sim \delta B r^{\delta-1}
\end{aligned}
$$

as $r \rightarrow 0+$, by the first part of Lemma 3. Setting $d=b$ and $n=1$ in Theorem 9 completes the argument.

Now let $a^{+}$denote the class of functions $f(z)$, analytic with $R(f) \geq 0$ in $y>0$, and assume that $0<a, b<\pi$ and that $-1<\delta<1$.

Theorem 12. Suppose that $f(z)$ is in $a^{+}$, that $(b-a) \delta \neq(\pi / 2) \bmod \pi$, and that

$$
\begin{aligned}
R\left\{f\left(r e^{i a}\right)\right\} & \sim A r^{\delta}, \\
I\left\{f\left(r e^{i b}\right)\right\} & \sim B r^{\delta}
\end{aligned}
$$

as $r \rightarrow 0+$. Then for all $0<\theta<\pi$ and $n \geq 1$,

$$
\begin{aligned}
f\left(r e^{i \theta}\right) & =f^{*}\left(r e^{i \theta}\right)+o\left(r^{\delta}\right), \\
\frac{d^{n}}{d z^{n}} f\left(r e^{i \theta}\right) & =\frac{d^{n}}{d z^{n}} f^{*}\left(r e^{i \theta}\right)+o\left(r^{\delta-n}\right)
\end{aligned}
$$

as $r \rightarrow 0+$, where

$$
f^{*}(z)=\frac{A e^{-i b \delta}+i B e^{-i a \delta}}{\cos (b-a) \delta} z^{\delta} .
$$

Proof. Set $u=R(f)$ and $v=I(f) ; u(z)$ is in $H^{+}$. By (5.4) we see that

$$
\begin{aligned}
& u\left(r e^{i a}\right) \sim A r^{\delta}, \\
& v\left(r e^{i b}\right) \sim B r^{\delta}
\end{aligned}
$$

as $r \rightarrow 0+$. With Theorem 7 and the second part of Lemma 3 we obtain

$$
\frac{\partial}{\partial r} v\left(r e^{i b}\right) \sim \delta B r^{\delta-1}
$$

as $r \rightarrow 0+$, and appealing to the Cauchy-Riemann equations we conclude that

$$
D_{d}^{1} u\left(r e^{i b}\right) \sim \delta B r^{\delta-1}
$$

as $r \rightarrow 0+$, where $d=b-\pi / 2$. Now apply Theorem 9 to the first part of (5.7) and to (5.8); we obtain (4.4) with

$$
\begin{aligned}
(1+\delta) C & =\frac{A \cos b \delta+B \sin a \delta}{\cos (b-a) \delta}, \\
(1+\delta) D & =\frac{A \cos (\pi-b) \delta-B \sin (\pi-a) \delta}{\cos (b-a) \delta} .
\end{aligned}
$$

From Theorem 4 it follows that for all $0<\theta<\pi, \psi$, and $n \geq 1$ 


$$
\begin{aligned}
u\left(r e^{i \theta}\right) & =u^{*}\left(r e^{i \theta}\right)+o\left(r^{\delta}\right), \\
D_{\psi}^{n} u\left(r e^{i \theta}\right) & =D_{\psi}^{n} u^{*}\left(r e^{i \theta}\right)+o\left(r^{\delta-n}\right)
\end{aligned}
$$

as $r \rightarrow 0+$, where $u^{*}=R\left(f^{*}\right)$ and $f^{*}(z)$ is as defined in (5.6). Set $v^{*}=I\left(f^{*}\right)$ and observe, by the Cauchy-Riemann equations and the second part of $(5.9)$, that

$$
\frac{\partial}{\partial \phi} v\left(r e^{i \phi}\right)=\frac{\partial}{\partial \phi} v^{*}\left(r e^{i \phi}\right)+o\left(r^{\delta}\right)
$$

as $r \rightarrow 0+$. We conclude $\left({ }^{3}\right)$ that for all $0<\theta<\pi$,

$$
\begin{aligned}
v\left(r e^{i \theta}\right) & =\int_{b}^{\theta} \frac{\partial}{\partial \phi} v\left(r e^{i \phi}\right) d \phi+v\left(r e^{i b}\right) \\
& =\int_{b}^{\theta} \frac{\partial}{\partial \phi} v^{*}\left(r e^{i \phi}\right) d \phi+v^{*}\left(r e^{i b}\right)+o\left(r^{\delta}\right) \\
& =v^{*}\left(r e^{i \theta}\right)+o\left(r^{\delta}\right)
\end{aligned}
$$

as $r \rightarrow 0+$ and the proof is complete.

Finally if $|f(z)| \leq M$ we can replace $f(z)$ by $f(z)+M$ and obtain the Lindelöf theorem from Theorem 12 with $a=b$ and $\delta=0$.

\section{REFERENCES}

1. A. C. Allen and E. R. Kerr, The converse of Fatou's theorem, J. London Math. Soc. vol. 28 (1953) pp. 80-89.

2. A. Erdélyi, Tables of integral transforms, vol. I, New York, 1954.

3. G. H. Hardy, Divergent series, Oxford, 1949.

4. L. H. Loomis, The converse of the Fatou theorem for positive harmonic functions, Trans. Amer. Math. Soc. vol. 53 (1943) pp. 239-250.

5. L. H. Loomis and D. V. Widder, The Poisson integral representation of functions which are positive and harmonic in a half-plane, Duke Math. J. vol. 9 (1942) pp. 643-645.

6. R. Nevanlinna, Eindeutige analytische Funktionen, Berlin, 1953.

7. S. Verblunsky, $A$ theorem on positive harmonic functions, Proc. Cambridge Philos. Soc. vol. 45 (1948) pp. 207-212.

8. D. V. Widder, The Laplace transform, Princeton, 1946.

UNIVERSITY OF MiCHIGAN, ANN ARBOR, Mich.

(3) It is not difficult to show that (5.10) holds uniformly in $\phi$ for $\phi$ between $b$ and $\theta$. 